\title{
Ulinastatin Promotes Regeneration of Peripheral Nerves After Sciatic Nerve Injury by Targeting let-7 microRNAs and Enhancing NGF Expression
}

This article was published in the following Dove Press journal:

Drug Design, Development and Therapy

\author{
Jincheng Zhang \\ Yingqi Zhang \\ Lei Chen \\ Zhitao Rao \\ Yeqing Sun (D)
}

Department of Orthopedics, Tongji Hospital, Tongji University School of Medicine, Shanghai, 200065, People's Republic of China
Correspondence: Yeqing Sun Department of OrthopedicsTongji Hospital,Tongji University School of Medicine, 389 Xincun Road, Putuo, Shanghai, 200065 People's Republic of China

Email leafsun306@I63.com
Background: Peripheral nerve injury is characterized as a common clinical problem. Ulinastatin (UTI) is a serine protease inhibitor with many biological activities including antiinflammatory and antioxidant effects. Nonetheless, it is unknown whether UTI has a protective effect on peripheral nerve injury.

Methods: Thirty rats were divided into the sham operation group, the sciatic nerve injury group (injected with normal saline), and the UTI treatment group $(80 \mathrm{mg} / \mathrm{kg} /$ day for two consecutive weeks). Sciatic nerve function index (SFI) was used to assess the biological functions of the sciatic nerve, and compound muscle action potential (CMAP) was measured by electrophysiology. The expressions of let-7 miRNA members were detected by quantitative real-time polymerase chain reaction (qRT-PCR). Nerve growth factor (NGF), nerve regeneration-related proteins GAP43 and NF200, and myelin formation-related proteins MAG and PMP22 expressions were explored by Western blot. After Schwann cells were transfected with let-7 mimics, pcDNA3.1-NGF, let-7 inhibitors, NGF siRNA and their corresponding controls, 5-ethynyl-2'-deoxyuridine (EdU) assay, and Transwell assays were employed to investigate the proliferation and migration of Schwann cells. $\mathrm{H}_{2} \mathrm{O}_{2}$ was utilized to construct oxidative injury to cells, and the contents of MDA, SOD, GSH, and CAT were determined.

Results: UTI treatment remarkably increased SFI of the rats and CMAP of sciatic nerve, enhanced nerve regeneration, and myelin regeneration, and raised the production of GAP43, NF200, MAG, and PMP22. Furthermore, it was found that UTI markedly reduced let-7 miRNAs' expressions and increased NGF expression after sciatic nerve injury. The dualluciferase reporter assay validated that let-7 miRNAs targeted NGF, and functional experiments demonstrated that low expression of let-7 miRNAs and NGF overexpression contributed to Schwann cells' proliferation and migration. Additionally, UTI treatment repressed the oxidative stress regulated by let-7/NGF axis.

Conclusion: UTI modulates the let-7/NGF axis to inhibit oxidative stress, promote nerve regeneration, and facilitate function recovery after peripheral nerve injury.

Keywords: ulinastatin, let-7 microRNAs, NGF, peripheral nerve injury

\section{Introduction}

Peripheral nerve injury is a common disease that causes degeneration of axons and their myelin sheaths at the distal end of the injury site, which seriously affects the life quality of the patients. ${ }^{1}$ Unlike the central nervous system, the peripheral nervous system possesses regenerative capabilities. Neural regeneration is a complicated biological phenomenon involving many cell types, growth factors, and different components of 
extracellular matrix. ${ }^{2}$ Schwann cells are the main glial cells in peripheral nervous system, and they attach great importance to peripheral nerve regeneration. ${ }^{3,4}$ After sciatic nerve injury, a series of molecular and cellular events occur, which contribute to Wallerian degeneration: in brief, the myelin sheath of nerves is disintegrated to granular and amorphous debris that cut off the distal stump of transected nerve, then the myelin sheath is transformed into short segments, and phagocytosis of the myelin debris and axon fibers is mediated by the activated macrophages, resulting in the degeneration of nerve stumps. ${ }^{5}$ Schwann cells can dedifferentiate, proliferate, and migrate to the site of injury, together with macrophages to eliminate myelin fragments, forming a Büngner band, which provides a condition for axon regeneration. ${ }^{6}$ Although the peripheral nervous system can spontaneously regenerate to some extent, its functional recovery is usually not satisfactory, especially in the case of severe injury. Therefore, novel and effective drugs are needed to promote the proliferate and migration of Schwann cells and facilitate neural regeneration.

Belonging to the family of neurotrophins, nerve growth factor (NGF) is involved in the development and maintenance of peripheral nervous system and central nervous system. ${ }^{7,8}$ Many previous researches suggest the beneficial effects of NGF on the regeneration of peripheral nervous system. $^{9-13}$ For example, NGF can improve the structural and functional recovery after sciatic nerve injury in rats. ${ }^{12}$ Moreover, NGF overexpression markedly improves Schwann cells-mediated axon regeneration during peripheral nerve repair. ${ }^{13}$ Nevertheless, its upstream regulatory mechanism has not yet been clarified.

MicroRNA (miRNA), a small non-coding RNA, whose length is approximately 20-23 nucleotides, inhibits the translation process by binding to the 3 '-untranslated region (3'UTR) of mRNA. Many miRNAs are reported to regulate neuron development, degeneration, and regeneration. ${ }^{14,15}$ For example, miR-7 regulates neural stem cell migration and proliferation through targeting cdc42 and represses peripheral nerve repair. ${ }^{16}$ As one of the most conservative members of the miRNAs family, let-7 draws much attention for its role in modulating organ development, internal environment homeostasis, and tumorigenesis. ${ }^{17}$ Additionally, it is reported that let-7 participates in the regulation of neuron fate, affecting neuron degeneration and regeneration. ${ }^{18-20}$ But the function of let-7 on the recovery of peripheral nerve injury is rarely reported.

Ulinastatin (UTI) is a serine protease inhibitor with a variety of biological activities, including antioxidant and anti-inflammatory activities, and is widely used to treat sepsis and pancreatitis. $^{21,22}$ It is reported that UTI treatment can increase NGF and brain-derived neurotrophic factor expression, and prevent oligodendrocyte apoptosis. $^{23}$ UTI also protects H9c2 cells from ischemia and hypoxia by inhibiting the NF- $\mathrm{B}$ pathway, reducing inflammation, attenuating oxidative stress, and repressing apoptosis. $^{24}$ However, the protective effect of UTI on peripheral nervous system remains unclear.

The main emphasis of this work lies in investigating the effects of UTI on peripheral nerves regeneration and functional recovery after peripheral nerve injury with a rat model of sciatic nerve injury. It was found that UTI remarkably suppressed let-7 expression and facilitated NGF expression. In vitro experiments showed that UTI enhanced the proliferation and migration of Schwann cells by modulating the let-7/NGF axis, alleviated the oxidative stress response, and thereby achieved its neuroprotective effect.

\section{Materials and Methods}

\section{Animals and Treatments}

Adult male Sprague-Dawley rats (180-220 g, 5-6 weeks old) were randomly divided into three groups: the sham group $(\mathrm{n}=10)$, the saline group (sciatic nerve injury model + intraperitoneal injection of equivalent saline, $\mathrm{n}=10$ ), and UTI group [sciatic nerve injury model + intraperitoneal injection of UTI $(80 \mathrm{mg} / \mathrm{kg} / \mathrm{day}$ for two consecutive weeks, $\mathrm{n}=10)]$. Rats were reserved in an environment with plenty of food and water and a light and dark cycle of $12 \mathrm{~h} / 12 \mathrm{~h}$. All experiments were performed in accordance with the NIH Guide for the Care and Use of Laboratory Animals. The research protocol was endorsed by the Ethics Committee of Tongji Hospital.

To establish sciatic nerve injury model, firstly, the rats were anesthetized by intraperitoneal injection of a compound anesthetic $(85 \mathrm{mg} / \mathrm{kg}$ acetaldehyde trihydrate monohydrate, $42 \mathrm{mg} / \mathrm{kg}$ magnesium sulfate, and $17 \mathrm{mg} / \mathrm{kg}$ sodium pentobarbital). After the left sciatic nerve was exposed, $1 \mathrm{~cm}$ long sciatic nerve was cut at the proximal site of the tibia and common peroneal nerve, followed by the suture of incision site. To reduce discomfort and possible mechanical stimuli, rats were housed in cages with sawdust-covered solid flooring after surgery. After $14 \mathrm{~d}$, the rats were decapitated and the sciatic nerve $(0.5 \mathrm{~cm}$ long) was obtained to prepare mRNA and protein samples. 


\section{Sciatic Nerve Function Index (SFI)}

SFI was employed to assess the function of the sciatic nerve according to a previous report. ${ }^{25}$ In brief, the hind paws of the rats were marked with black ink, and the rats ( $n=4$ /group) were released to walk along a corridor of $6 \times$ 60 square centimeters covered with white paper. Then, according to the footprints of rats on white paper, SFI = -38.3 [EPL (experimental podogram length) - NPL (normal podogram length)]/NPL + 109.5[ETW (experimental toes width) - NTW (normal toes width)]/NTW + 13.3[EIT (experimental inter-toe distance) - NIT (normal inter-toe distance)]/NIT - 8.8. An SFI value of -100 indicated complete loss of function, while an SFI value of 0 illustrated normal function.

\section{Electrophysiological Analysis}

Compound muscle action potential (CMAP) was used to reflect the number and functional status of excitable cells. After anesthesia, the sciatic nerve was exposed and the nerve repair site was identified under a surgical microscope. A rubber dam was used to isolate the nerve repair area from the surrounding muscles. A bipolar electrode for stimulation was placed $10 \mathrm{~mm}$ proximal to the injury site of sciatic nerve. A recording electrode was placed in the gastrocnemius muscle to record CMAP peak amplitude and CMAP latency.

\section{Cell Culture and Processing}

Primary Schwann cells were isolated from the sciatic nerve of newborn 3- to 4-d-old newborn rats. To remove connective tissues and fibroblasts, the tissues were dissociated by incubating them in Dulbecco's modified eagle's medium (DMEM; Invitrogen, Carlsbad, CA, USA) supplemented with $0.25 \%$ trypsin and $0.1 \%$ collagenase type A (Sigma, St Louis, MO, USA) for $20 \mathrm{~min}$ at $37^{\circ} \mathrm{C}$. After that, the mixtures were centrifuged, placed onto tissue culture flasks pre-coated with poly-L-lysine (Sigma, St Louis, MO, USA) and then cultured in DMEM added with $4 \mathrm{mM}$ glutamine (Sigma, St Louis, MO, USA), 10\% fetal bovine serum (FBS; Gibco, Carlsbad, CA, USA), and $100 \mathrm{U} / \mathrm{mL}$ penicillin and $100 \mu \mathrm{g} / \mathrm{mL}$ streptomycin (Gibco, Carlsbad, CA, USA). 2 $\mathrm{d}$ later, $10^{-5} \mathrm{M}$ cytosine arabinoside (Sigma, St Louis, MO, USA) was used to remove the contaminating fibroblasts. Then, the Schwann cells were further purified with antiThy1.1 monoclonal antibody and Low-Tox ${ }^{\circledR}-\mathrm{M}$ rabbit complement (Cedarlane, Burlington, NC, USA). The collected Schwann cells were cultured in DMEM containing 10\% FBS. To stimulate the Schwann cells growth, $5 \mu \mathrm{M}$ forskolin and $4.1 \mu \mathrm{g} / \mathrm{mL}$ insulin (Sigma, St Louis, MO, USA) were added. The purified cells were then transplanted into a 6-well plate for $24 \mathrm{~h}$. Conforming to the instructions, the Schwann cells were transfected with let-7 mimics, pcDNA3.1-NGF, let-7 inhibitors, NGF siRNA, and their corresponding controls (Ribobio, Guangzhou, China) using Lipofectamine ${ }^{\circledR}$ 3000 reagent (Invitrogen, Carlsbad, CA, USA).

Schwann cells were treated with $\mathrm{H}_{2} \mathrm{O}_{2}$ to simulate oxidative damage. $\mathrm{H}_{2} \mathrm{O}_{2}$ stimulation group was cultured in DMEM containing $100 \mu \mathrm{mol} / \mathrm{L} \mathrm{H}_{2} \mathrm{O}_{2}$ for $4 \mathrm{~h}$. In the $\mathrm{H}_{2} \mathrm{O}_{2}+$ UTI treatment group, UTI $(100 \mathrm{U} / \mathrm{mL})$ treatment was supplemented $2 \mathrm{~h}$ before the addition of $\mathrm{H}_{2} \mathrm{O}_{2}$.

\section{Quantitative Real-Time Polymerase Chain Reaction (qRT-PCR)}

To detect mRNA expression of NGF, total RNA was extracted employing TRIzol reagent (Invitrogen, Carlsbad, CA, USA). RNA was reversely transcribed into cDNA using Prime-Script RT reagent Kit (TaKaRa, Dalian, China). To determine let-7 expression, RNA was reversely transcribed utilizing the TaqMan MicroRNA Reverse Transcription Kit (Applied Biosystems, Foster City, CA, USA) and Stem-Loop miRNA qPCR Primer (Hairui, Guangzhou, China) following the manufacturer's instructions. qRT-PCR was performed on an Applied Biosystems StepOne Real-time PCR System using SYBR Premix Ex Taq kit (TaKaRa, Dalian, China) according to the manufacturer's instructions. Each experiment is repeated three times. Glyceraldehyde-3-phosphate dehydrogenase (GAPDH) and U6 were used as the internal references for NGF and let-7 miRNAs, respectively, and the relative expression was calculated employing the $2^{-\Delta \Delta \mathrm{Ct}}$ method. The sequences of the NGF primers were listed as follows: forward: 5'-CCAAGGACGCAGCTTTCTATC-3', reverse: 5'-CTGTGTCAAGGGAATGCTGAAG-3'; GAPDH primer: forward: 5'-GGAGCGAGATCCCTCCAAAAT-3', reverse: 5'-GGCT GTTGTCATACTTCTCATGG-3'.

\section{Western Blot}

Schwann cells and sciatic nerve were lysed with RIPA lysis buffer (Beyotime, Shanghai, China), and the quantity of the protein in the lysate was quantified using the BCA protein quantification kit (Beyotime Biotechnology, Shanghai, China). After loading buffer was added, the extracted protein was denatured in boiling water, and then the protein samples were separated by $10 \%$ SDS-PAGE and then transferred to a PVDF membrane (Millipore, Bedford, MA, USA). Then, these membranes were blocked with 5\% skim milk at room 
temperature for $30 \mathrm{~min}$, and primary antibodies, including anti-NGF (Abcam; 1:1000; ab6199), anti-myelin-associated glycoprotein (MAG) (Abcam; 1:1000; ab89780), antiperipheral myelin protein-22 (PMP22) (Abcam; 1:1000; ab270400), anti-growth-associated protein-43 (GAP43) (Abcam; 1:1000; ab16053), anti-neurofilament 200 (NF200) (Abcam; 1:1000; ab134306), and anti- $\beta$-actin (Abcam 1:3000; ab8227) were added to incubate the membranes at $4^{\circ} \mathrm{C}$ overnight. The next day, after being washed twice with TBST, the membranes were incubated with the HRP-conjugated secondary antibody for $1 \mathrm{~h}$ at room temperature. The membrane was rinsed for 3 times before the protein bands were visualized with enhanced chemiluminescence solution (Thermo Fisher Scientific, Inc., Carlsbad, CA, USA) exposed on film.

\section{Cell Proliferation Experiment}

Thymidine analog EdU (5-ethynyl-2'-deoxyuridine) can be readily incorporated into DNA during synthesis and was used to detect Schwann cells proliferation. The cultured Schwann cells were suspended in medium and seeded at $1 \times 10^{5}$ cells/well into a 96-well plate pre-coated with $0.01 \%$ polylysine. Schwann cells were incubated with $50 \mu \mathrm{M}$ EdU solution (Ribobio, Guangzhou, China) for $12 \mathrm{~h}$. Subsequently, cells were fixed with 4\% paraformaldehyde and stained with Apollo staining solution and Hoechst 33,342 (Riboio, Guangzhou, China) for $30 \mathrm{~min}$. Then, fluorescence microscopy (Leica, Bensheim, Germany) was used to observe the cells in 5 randomly selected visual fields, and then the ratio of EdU positive cells to total cells was calculated.

\section{Cell Migration Experiment}

The migration of Schwann cells was measured employing Transwell system ( $8 \mu \mathrm{m}$ pore size, Corning, Beijing, China) which was coated with a layer of fibronectin. A total of 100 $\mu \mathrm{L}$ of suspended Schwann cells $\left(1 \times 10^{5}\right.$ cells $\left./ \mathrm{mL}\right)$ was seeded into the upper chamber, and $600 \mu \mathrm{L}$ medium was added to the lower chamber. After being incubated in an incubator for $24 \mathrm{~h}$, Schwann cells in the upper chamber were wiped off, and Schwann cells passing through the membrane were fixed with formaldehyde solution and stained with $0.1 \%$ crystal violet solution. Finally, the cells were observed and counted utilizing an inverted microscope.

\section{Luciferase Reporter Experiment}

The NGF 3'-UTR sequence was amplified from the genomic DNA by PCR and subcloned into the luciferase reporter vector downstream of the stop codon of the luciferase gene. Overlap PCR was used to construct a 3'-UTR mutation reporter plasmid. The sequences of the wild-type and mutant 3'UTRs were confirmed by sequencing. A mixture of $30 \mathrm{ng}$ reporter plasmids, 5 pmol miRNA mimic, and 5 ng plasmid carrying Renilla luciferase gene was co-transfected into HEK293T cells using Lipofectamine ${ }^{\circledR} \quad 3000$ reagent (Invitrogen, Carlsbad, CA, USA). After $48 \mathrm{~h}$ of incubation, relative luciferase activity was analyzed according to the instructions Dual-Luciferase Reporter Assay System (Promega, Madison, WI, USA).

\section{Lipid Peroxidation Determination}

Twenty-four hours after incubation with UTI, the malondialdehyde (MDA) level of Schwann cells in each group was measured to assess lipid peroxidation. Briefly, the MDA in the sample was reacted with thiobarbituric acid (Solarbio, Beijing, China) at high temperature $\left(90-100^{\circ} \mathrm{C}\right)$. The MDA-TAB compound was then measured at $450 \mathrm{~nm}$ with a spectrophotometer.

\section{Determination of Antioxidant Enzyme Activity}

Superoxide dismutase (SOD) and catalase (CAT) activities of Schwann cells in each group were detected $24 \mathrm{~h}$ after incubation with UTI to evaluate their antioxidant enzyme activities. SOD activity was examined by xanthine oxidase method (Solarbio, Beijing, China). Hydrogen peroxide method was applied to investigate the CAT activity (Solarbio, Beijing, China).

\section{GSH Determination}

After $24 \mathrm{~h}$ of incubation with UTI, glutathione (GSH) level in Schwann cells was measured with GSH detection kit (Amersham Biosciences, USA) according to the protocol provided by the manufacturer.

\section{Statistical Analysis}

All statistical analyses were performed using SPSS version 17.0 software (SPSS Inc., Chicago, IL, USA). All data were expressed as mean \pm standard error of mean (SEM). Students' $t$-test was used for statistical analysis between groups. $P<0.05$ signified statistical significance.

\section{Result}

\section{UTI Improved Motor Function Recovery of Rats from Sciatic Nerve Injury}

SFI was employed to assess the impact of UTI on the recovery of motor function of rats after sciatic nerve injury. The 
results revealed a gradual increase in SFI among the rats in the saline group, indicating a spontaneous recovery of motor function. In the meantime, the SFI of rats in the UTI group also gradually increased, and the SFI of the UTI treatment group was notably higher than that of the saline group (Figure 1A; $n=4 /$ group). Furthermore, we used the CMAP to analyze the function of sciatic nerve after $14 \mathrm{~d}$ of sciatic nerve injury in rats. As was shown, UTI treatment increased the amplitude, but reduced the latency of CMAP (Figure 1B and $C ; n=3$ /group). These data indicated that after sciatic nerve injury in rats, UTI treatment facilitated the regeneration of sciatic nerve and improved the functional recovery.

\section{UTI Was Beneficial for Myelinogenesis and Nerve Regeneration}

To probe the effect of UTI on myelinogenesis and nerve regeneration after sciatic nerve injury, Western blot was employed to detect changes in MAG and PMP22 expressions, the findings of which demonstrated that compared with the sham group, MAG and PMP22 expressions decreased in the saline group, indicating that the structure and function of the myelin sheath were injured; compared to saline group, UTI treatment promoted $\mathrm{MAG}$ and PMP22 expressions (Figure $2 \mathrm{~A}$ and $\mathrm{B} ; \mathrm{n}=3$ /group). Western blot also revealed that GAP43 expression in the saline group increased in comparison with the sham group after sciatic nerve injury, while it was lower than in UTI group; however, in contrast with the sham group, NF200 expression was remarkably reduced in the saline group, and NF200 expression was remarkably increased in UTI group compared with the saline group (Figure 2C and D; $\mathrm{n}=3$ /group). Based on these data, we inferred that UTI enhanced myelinogenesis and nerve regeneration after sciatic nerve injury.

\section{The Expression of let-7 miRNAs Was Regulated by UTI}

To explore the mechanism by which UTI ameliorated sciatic nerve injury in rats, the expression of let-7 miRNAs in sciatic nerve was detected using qRT-PCR, and the results revealed that compared with the sham group, let-7a, let-7d, let-7i, and miR-98 in the saline group were markedly up-regulated, let-7c and let-7f were remarkably down-regulated, and let- $7 \mathrm{~b}$ and let-7e expressions were not significantly affected (Figure 3A-H; $n=3$ / group). However, compared with the saline group, all of the eight members of the let-7 family were notably lower in the UTI group (Figure 3A-H; $\mathrm{n}=3$ /group). The data implied that UTI attenuated peripheral nerve injury, and enhanced myelin sheath and nerve regeneration by regulating the expressions of let-7 miRNAs.

\section{NGF Was a Target Gene of let-7 miRNAs}

According to a previous report, let-7 miRNAs can target NGF. ${ }^{26}$ Consistently, this study demonstrated that the targeting relationship was predicted by TargetScan databases (Figure 4A). Dual-luciferase reporter assay showed that when wild-type NGF reporter and let-7d mimics were cotransfected into HEK293T cells, the luciferase activity was remarkably reduced, but let-7d mimics did not reduce the luciferase activity of mutant NGF reporter, which validated the binding site (Figure 4B). Then, we used qRT-PCR and Western blot to detect NGF mRNA and protein expressions in the sciatic nerve of rats from each group. The results revealed that in comparison with the sham group, NGF mRNA and protein expressions were remarkably increased in saline group and UTI group, and UTI induced a higher expression of NGF compared with saline (Figure 4C and D; $\mathrm{n}=3$ /group). Additionally, the transfection of let-7 mimics
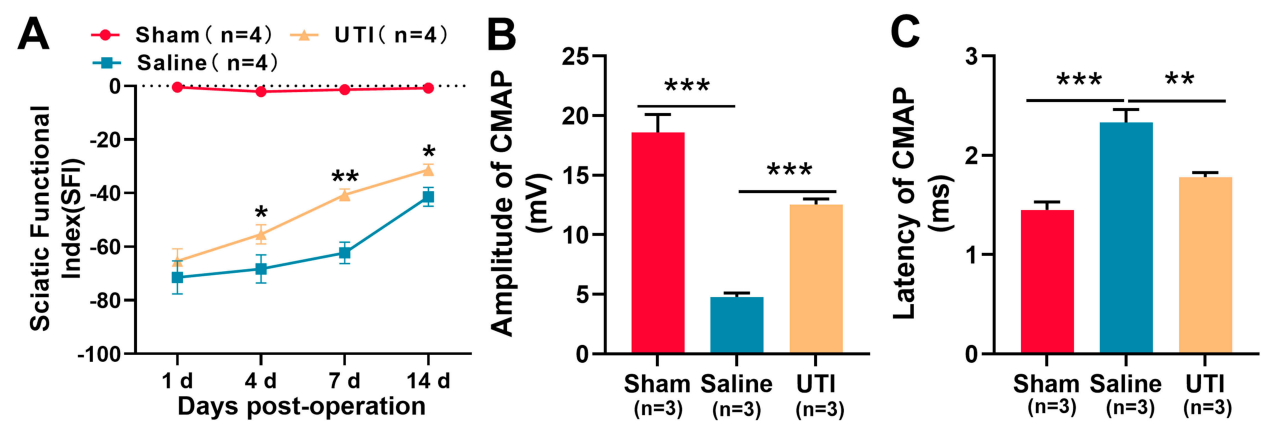

Figure I UTI accelerated function recovery of nerves after sciatic nerve injury. (A) SFI method was used to evaluate the function recovery after sciatic nerve injury, $n=4 /$ group. (B and C) UTI treatment significantly affected the amplitude $(\mathbf{B})$ and latency $(\mathbf{C})$ of CMAP, $\mathrm{n}=3 /$ group. $* \mathrm{P}<0.05$, $* * \mathrm{P}<0.0 \mathrm{I}$, and $* * * \mathrm{P}<0.00 \mathrm{I}$.

Abbreviations: UTI, ulinastatin; SFI, sciatic functional index; CAMP, compound muscle action potential; Sham, sham group; Saline, saline-treated group; UTI, UTI-treated group. 

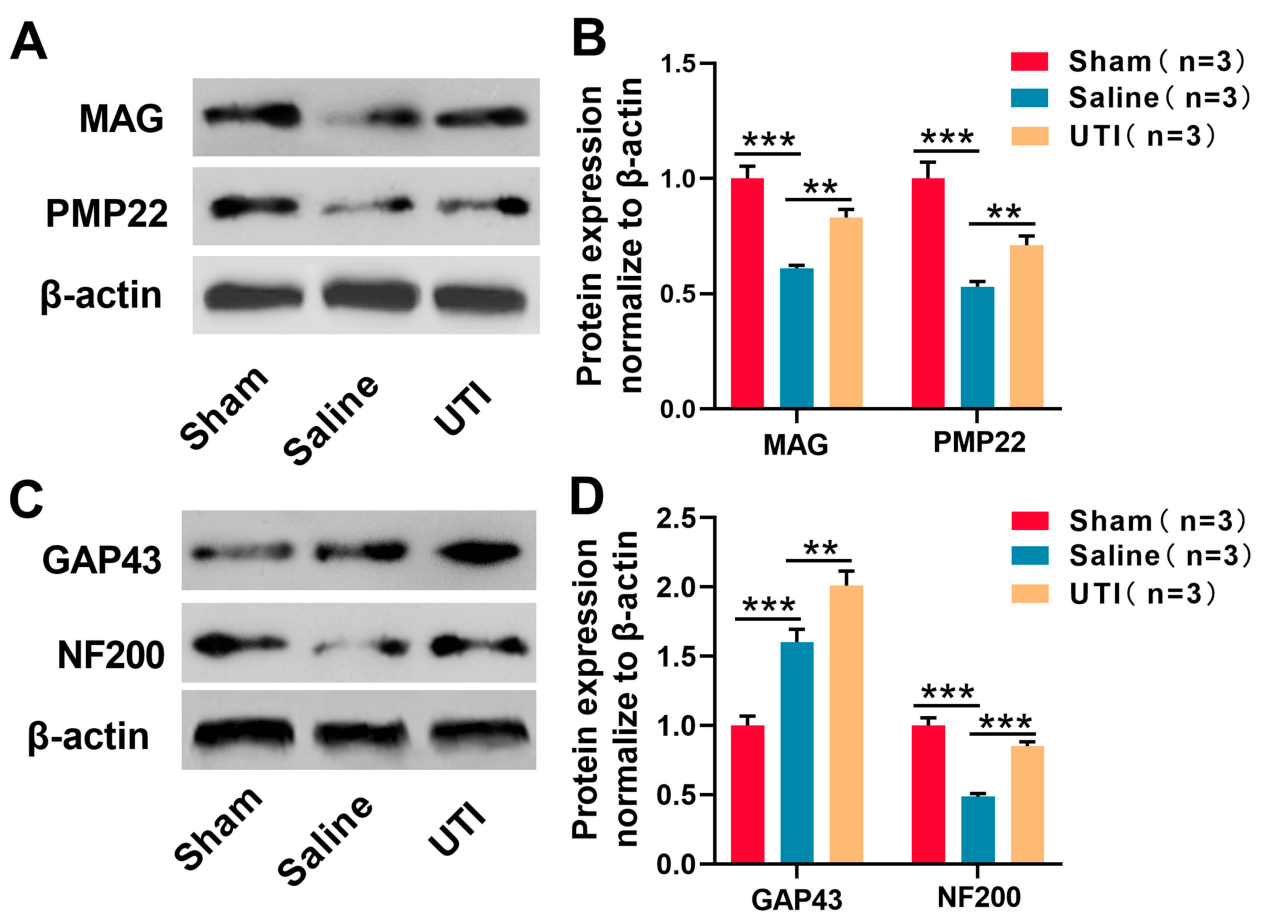

Figure 2 UTI was beneficial for myelinogenesis and nerve regeneration after sciatic nerve injury. (A) and (B) MAG and PMP22 expressions were analyzed by Western blot, $\mathrm{n}=3$ /group. (C and D) GAP43 and NF200 expressions were analyzed by Western blot, $\mathrm{n}=3$ /group. $* * P<0.01$, and $* * * P<0.001$.

Abbreviations: MAG, myelin-associated glycoprotein; PMP22, peripheral myelin protein 22; GAP43, growth-associated protein 43; NF200, neurofilament 200; Sham, sham group; Saline, saline-treated group; UTI, UTI treated group.
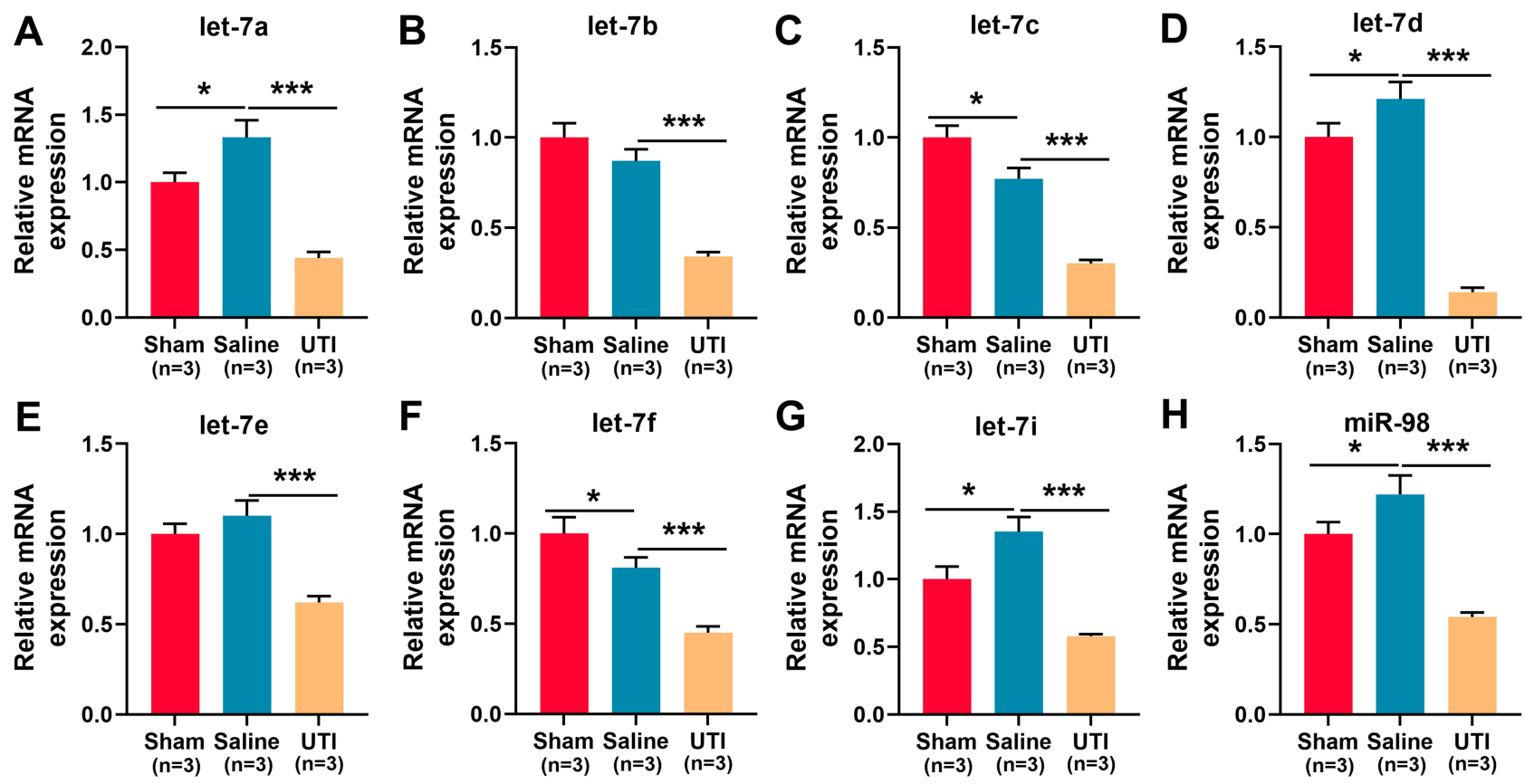

Figure 3 Expressions of let-7 family members in the sciatic nerve of rats after sciatic nerve injury. (A-H) qRT-PCR was used to detect the relative expression levels of eight members of let-7 family: (A) let-7a; (B) let-7b; (C) let-7c; (D) let-7d; (E) let-7e; (F) let-7f; (G) let-7i; (H) miR-98, $\mathrm{n}=3 /$ group. $* P<0.05$, and *** $<<0.00$ I. Abbreviations: Sham, sham group; Saline, saline-treated group; UTI, UTI-treated group. 

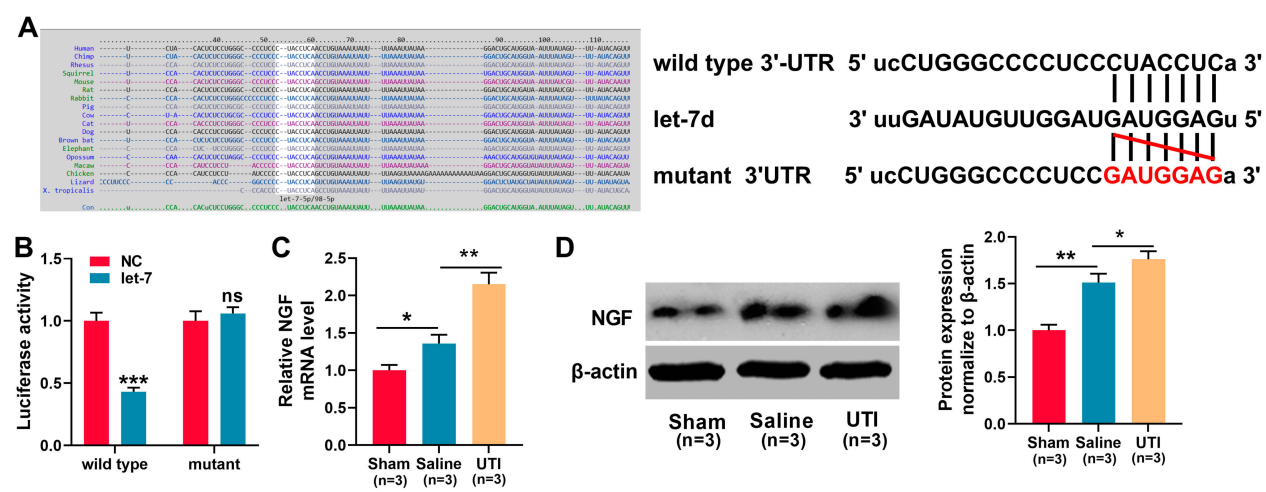

Figure 4 NGF was a target gene of let-7. (A)TargetScan databases was employed to predict the target sites between let-7 microRNAs and NGF, and NGF wild-type and mutant reporter vectors were constructed. (B) Dual-luciferase reporter gene experiments were used to verify the targeting relationship between let-7 and the $3^{\prime} U T R$ of NGF. (C-D) qRT-PCR and Western blot were used to detect the expression levels of NGF mRNA and protein in the sciatic nerve of rats, $\mathrm{n}=3 /$ group. $* P<0.05$, $* * P<0.0$ I, and $* * * P<0.001$

Abbreviations: Wild type, wild type of NGF 3'UTR; mutant, mutant of NGF 3'UTR; NC, negative control; let-7, let-7 mimic; NGF, nerve growth factor; Sham, sham group; Saline, saline-treated group; UTI, UTI-treated group; ns, not significant.

into Schwann cells significantly reduced the expression of NGF (Figure 5A). Collectively, these results indicated that NGF was a target gene of let-7 in Schwann cells.

\section{Let-7 Regulated the Migration and Proliferation of Schwann Cells Through NGF}

To further investigate whether let-7 regulated Schwann cells' migration and proliferation via NGF, let-7 mimics and
pcDNA3.1-NGF were co-transfected into Schwann cells. Western blot revealed that let-7 observably reduced NGF protein expression, and co-transfection of pcDNA3.1-NGF restores the expression of NGF (Figure 5A). As was shown, compared with control group, the migration and proliferation of let-7 mimics-transfected Schwann cells were remarkably reduced, while co-transfection of pcDNA3.1-NGF into Schwann cells reversed the reduction in migration and proliferation (Figure 5B and C). Additionally, let-7 inhibitors and NGF siRNA were co-transfected into Schwann cells, and
A

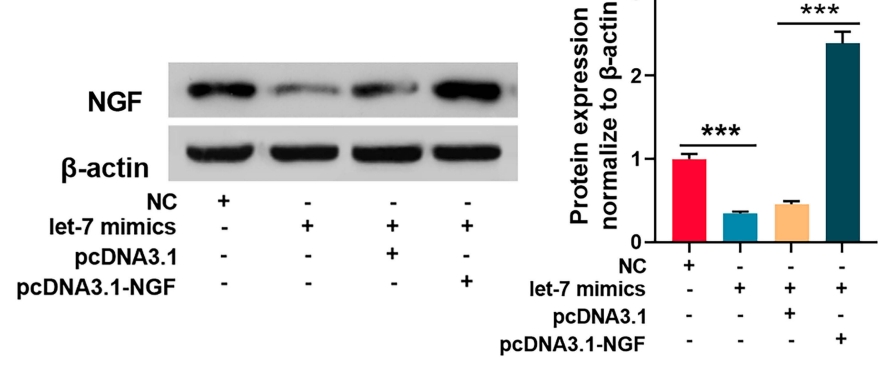

D

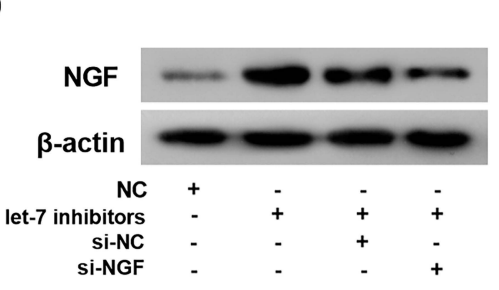

B

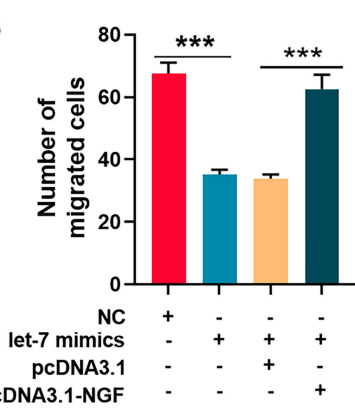

E

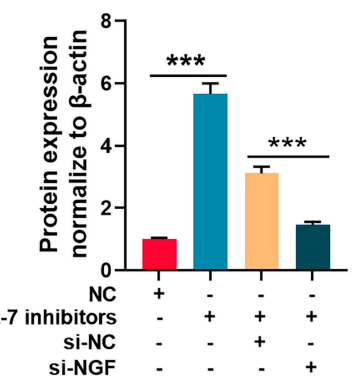

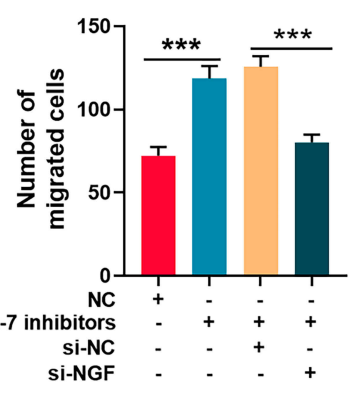

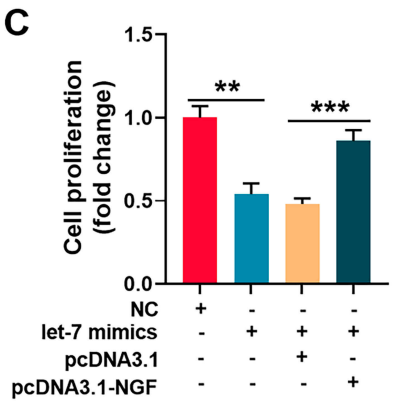

$\mathbf{F}$

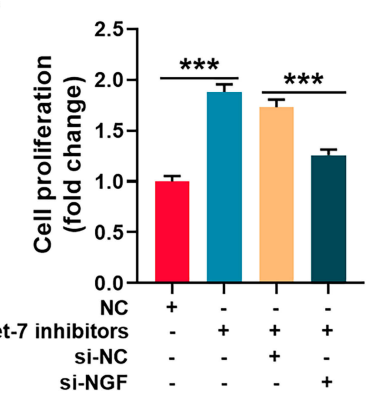

Figure 5 Let-7 regulated the migration and proliferation of Schwann cells through NGF. (A) Let-7 mimics and pcDNA3. I-NGF were transfected into Schwann cells, and Western blot was used to detect the expression of NGF protein. (B and $\mathbf{C})$ The migration (B) and proliferation (C) of Schwann cells were detected by Transwell assay and EdU assay respectively. (D) Let-7 inhibitors and NGF siRNA were transfected into Schwann cells, and Western blot was used to detect the expression of NGF protein. $(\mathbf{E}$ and $\mathbf{F})$ The migration $(\mathbf{E})$ and proliferation $(\mathbf{F})$ of Schwann cells were detected by Transwell assay and EdU assay respectively. $* * P<0.01$, and $* * * P<0.00$ I.

Abbreviation: NGF, nerve growth factor. 
the result illustrated that let-7 inhibitors significantly upregulated the expression of NGF, and co-transfection of NGF siRNA partly abolish this effect (Figure 5D). Furthermore, let-7 inhibitors facilitated SC migration and proliferation, while NGF siRNA reversed the increase in migration and proliferation (Figure $5 \mathrm{E}$ and $\mathrm{F}$ ). The data further validated that NGF was a downstream target gene of let-7, and demonstrated that let-7 regulated the migration and proliferation of Schwann cells through NGF.

\section{UTI Repressed Sciatic Nerve Injury-Induced Oxidative Stress}

Oxidative stress is one of the main cause of nerve injury and has a negative effect on the recovery of nerve function after peripheral nerve injury. To investigate the effect of UTI on oxidative stress after sciatic nerve injury. Schwann cells treated with $\mathrm{H}_{2} \mathrm{O}_{2}$ were employed to establish oxidative injury model, which was evaluated by measuring MDA, SOD, GSH, and CAT levels in Schwann cells. The content of MDA in $\mathrm{H}_{2} \mathrm{O}_{2}$-treated Schwann cells was remarkably higher than that of control group, indicating that lipid peroxidation of Schwann cells was increased by $\mathrm{H}_{2} \mathrm{O}_{2}$; when the $\mathrm{H}_{2} \mathrm{O}_{2}$-treated Schwann cells were incubated with UTI, the MDA content decreased markedly, implying that UTI remarkably inhibited lipid peroxidation of Schwann cells (Figure 6A). Additionally, the MDA content in Schwann cells transfected with let-7 inhibitors was decreased, and the MDA content in Schwann cells transfected with NGF siRNA was increased remarkably (Figure 6A). SOD activity, CAT activity, and GSH level were also detected to evaluate the oxidative injury of Schwann cells induced by $\mathrm{H}_{2} \mathrm{O}_{2}$. The data indicated that $\mathrm{H}_{2} \mathrm{O}_{2}$ markedly reduced the SOD activity, CAT activity, and GSH expression level of Schwann cells; after $\mathrm{H}_{2} \mathrm{O}_{2}$ treated Schwann cells were incubated with UTI, SOD, CAT activity, and GSH expression levels were remarkably increased. Further analysis revealed that the SOD activity, CAT activity, and GSH expression level in Schwann cells after transfection of let-7 inhibitors were notably higher than those of control group; nonetheless, SOD activity, CAT activity, and GSH expression levels were remarkably repressed in NGF siRNA transfected cells (Figure 6B-D). Collectively, these results suggested that UTI repressed oxidative stress-induced lipid peroxidation and injury of Schwann cells, and this effect was mediated by let-7/NGF axis.

\section{Discussion}

Peripheral nerve injury is considered as a global clinical problem, which is responsible for a huge medical and economic burden. ${ }^{27}$ Recently, many drugs and treatment strategies have been developed to improve the prognosis of peripheral nerve injury. ${ }^{28-30}$ UTI inhibits polymorphonuclear leukocyte-derived elastase and pro-inflammatory cytokines including TNF- $\alpha$, IL-1, IL-6, and IL-8; it also suppresses the activation of polymorphonuclear leukocyte, macrophages, and platelets, which mediates the injury of organs and tissues. $^{31}$ In some countries, UTI is applied to the clinical treatment of shock and pancreatitis, ${ }^{31}$ and accumulating basic medical researches suggests that it is promising to protect the organs and tissues from multiple pathogenic factors. $^{21-24}$ In the current study, it was found that UTI remarkably increased the SFI and amplitude of CMAP, but reduced the latency of CMAP, suggesting UTI improved regeneration and functional recovery of sciatic nerve after injury. The data highlighted the therapeutic value of UTI as a neuroprotective drug for peripheral nerve repair.

It is well known that neuron proliferation and remyelination after sciatic nerve injury are essential processes for rebuilding the functional nerves that innervate their corresponding target tissues. ${ }^{32}$ In the present study, we evaluated the regulatory functions of UTI on the expression levels of nerve growth-related proteins (GAP43 and NF200) and myelin formation-related proteins (MAG and PMP22). GAP43 is a protein kinase $\mathrm{C}$-activated phosphoprotein, which is involved in the regulation of axonal plasticity and regeneration. ${ }^{33,34}$ Neurofilaments are crucial in maintaining the structure and function of neurons, and disruption of neurofilament organization and expression or metabolism of neurofilament proteins is characteristic of neuropathy and nerve injury. ${ }^{35}$ MAG is a type I membrane protein and a member of the immunoglobulin superfamily; it participates in the maintenance of myelin sheath by functioning as adhesion molecule that mediates interactions between myelinating cells and neurons. ${ }^{36}$ PMP22 is mainly expressed by Schwann cells and takes part in the formation and maintenance of myelin sheath; importantly, PMP22 is crucial in protecting nerves from physical pressure, helping restore the structure after being pinched or squeezed. ${ }^{37,38}$ For example, isoquercitrin treatment significantly increases the production of GAP43, NF200, MAG, and PMP22, and promotes the nerve regeneration and remyelination. ${ }^{39}$ Our results revealed that UTI administration increased the expression of these markers, which suggested that UTI promoted the functional 

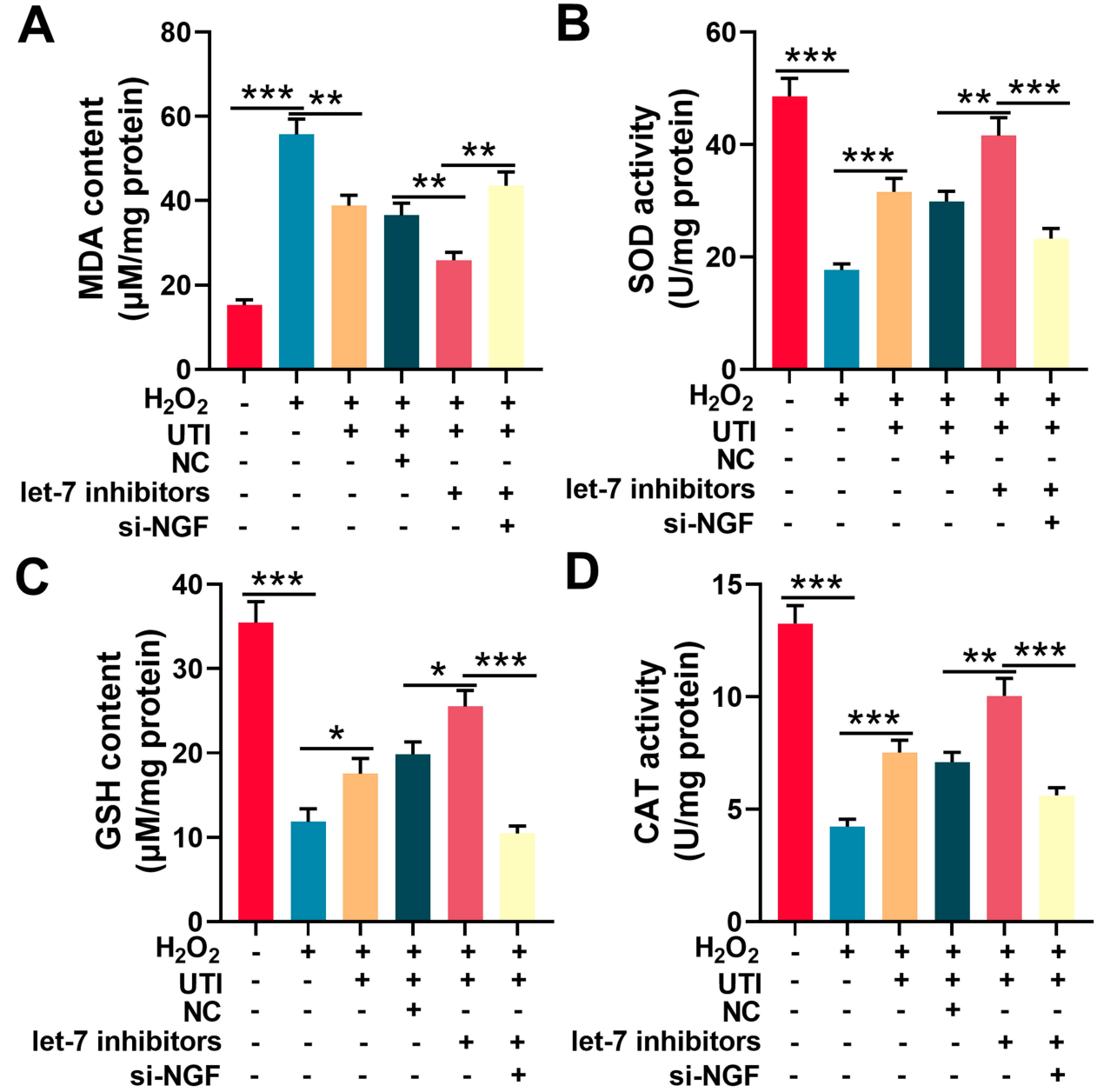

Figure 6 UTI repressed oxidative stress of Schwann cells induced by $\mathrm{H}_{2} \mathrm{O}_{2}$. MDA level (A), SOD activity (B), GSH level (C) and CAT activity (D) were detected with corresponding kit respectively after Schwann cells were incubated with $\mathrm{H}_{2} \mathrm{O}_{2}$. $* P<0.05$, $* * P<0.0 \mathrm{I}$, and $* * * P<0.00 \mathrm{I}$.

Abbreviations: UTI, ulinastatin; si-NGF, NGF siRNA; MDA, malondialdehyde; SOD, superoxide dismutase; GSH, glutathione; CAT, catalase.

recovery of peripheral nerve by facilitating the axonal outgrowth and remyelination.

MiRNAs are involved in modulating a variety of biological processes. ${ }^{40,41}$ For example, miR-192-5p inhibition can up-regulate the expression of XIAP, decrease the apoptosis of neurons, and promote the recovery of sensory function and the neuronal recovery and regeneration in rats with sciatic nerve injury. ${ }^{42}$ A previous research shows that let-7 miRNAs are involved in regulating the repair process of the sciatic nerve. ${ }^{43}$ The present study investigated the expression of eight members of let-7 family (including let7a, b, c, d, e, f, i, and miR-98) after sciatic nerve injury and UTI treatment in rats, and found that UTI remarkably repressed let-7 miRNAs expression. It is reported that let-7 inhibition promotes Schwann cells migration and axon outgrowth within a regenerative microenvironment by directly targeting NGF in vivo. ${ }^{26}$ It is reported that the proliferated Schwann cells not only secrete neurotrophic factors to promote nerve regeneration but also can migrate to the damaged site to promote regeneration of myelin sheath after peripheral nerve injury. ${ }^{26,44,45}$ The present study also investigated that whether let-7/NGF axis regulated the proliferation and migration of Schwann cells. Consistent with the previous report, ${ }^{26}$ it was demonstrated that inhibition of let-7 and NGF overexpression markedly enhanced the proliferation and migration of Schwann cells. Importantly, our data indicated that UTI treatment increased the expression of NGF in the sciatic nerve of rats. Collectively, the present work implied that UTI was beneficial to Schwann cells' function by modulating let-7/ NGF axis after peripheral nerve injury.

Peripheral nerve injury is often accompanied by oxidative stress and inflammatory responses. ${ }^{46,47}$ Peripheral nerve injury induces the production of reactive oxygen 
species and nitric oxide in axotomized neurons; additionally, Schwann cells and macrophages express proinflammatory molecules such as interleukin IL-1 $\beta$, IL-6, IL-12, TNF- $\alpha$, TGF- $\beta$, and in turn contribute to injury of neurons. $^{48,49}$ Research suggests that blocking oxidative stress can accelerate the repair process and facilitate functional recovery after peripheral nerve injury. ${ }^{50,51}$ In this research, $\mathrm{H}_{2} \mathrm{O}_{2}$ treatment was used to mimic oxidative stress to Schwann cells. The results suggested that UTI remarkably reduced the content of MDA and promoted the increase of activities of antioxidant SOD, CAT, and GSH. These data further revealed that UTI could probably ameliorate neuron injury and promote the regeneration of nerves by attenuating after oxidative stress.

In summary, this study indicates that UTI regulates let7/NGF axis, and represses oxidative stress, which can promote myelinogenesis, nerve regeneration, and functional recovery of nerves after peripheral nerve injury. This work provides molecular regulation mechanism for UTI as a potential neuroprotective drug and clues for the treatment of peripheral nerve injury.

\section{Data Sharing Statement}

The data used to support the findings of this study are available from the corresponding author upon request.

\section{Funding}

This study is supported by Shanghai Shenkang Hospital Development Center Clinical Research cultivation project (NO. SHDC12017X15.

\section{Disclosure}

The authors declare that they have no competing interests in this work.

\section{References}

1. Willand MP, Nguyen MA, Borschel GH, Gordon T. Electrical stimulation to promote peripheral nerve regeneration. Neurorehabil Neural Repair. 2016;30(5):490-496. doi:10.1177/154596831 5604399

2. Gu X, Ding F, Yang Y, Liu J. Construction of tissue engineered nerve grafts and their application in peripheral nerve regeneration. Prog Neurobiol. 2011;93(2):204-230. doi:10.1016/j.pneurobio.2010.11.002

3. Raphael AR, Perlin JR, Talbot WS. Schwann cells reposition a peripheral nerve to isolate it from postembryonic remodeling of its targets. Development. 2010;137(21):3643-3649. doi:10.1242/dev.05 7521

4. Chen YY, McDonald D, Cheng C, Magnowski B, Durand J, Zochodne DW. Axon and Schwann cell partnership during nerve regrowth. J Neuropathol Exp Neurol. 2005;64(7):613-622. doi:10.10 97/01.jnen.0000171650.94341.46
5. Amani H, Kazerooni H, Hassanpoor H, Akbarzadeh A, PazokiToroudi H. Tailoring synthetic polymeric biomaterials towards nerve tissue engineering: a review. Artif Cells Nanomed Biotechnol. 2019;47(1):3524-3539. doi:10.1080/21691401.2019.1639723

6. Jessen KR, Mirsky R. The success and failure of the Schwann cell response to nerve injury. Front Cell Neurosci. 2019;13:33. doi:10.3389/fncel.2019.00033

7. Aloe L, Rocco ML, Bianchi P, Manni L. Nerve growth factor: from the early discoveries to the potential clinical use. J Transl Med. 2012;10(1):239. doi:10.1186/1479-5876-10-239

8. Wood MD, MacEwan MR, French AR, et al. Fibrin matrices with affinity-based delivery systems and neurotrophic factors promote functional nerve regeneration. Biotechnol Bioeng. 2010;106 (6):970-979. doi:10.1002/bit.22766

9. Fiore M, Mancinelli R, Aloe L, et al. Hepatocyte growth factor, vascular endothelial growth factor, glial cell-derived neurotrophic factor and nerve growth factor are differentially affected by early chronic ethanol or red wine intake. Toxicol Lett. 2009;188 (3):208-213. doi:10.1016/j.toxlet.2009.04.013

10. Manni L, Rocco ML, Bianchi P, et al. Nerve growth factor: basic studies and possible therapeutic applications. Growth Factors. 2013;31(4):115-122. doi:10.3109/08977194.2013.804073

11. da Silva JT, Santos FM, Giardini AC, et al. Neural mobilization promotes nerve regeneration by nerve growth factor and myelin protein zero increased after sciatic nerve injury. Growth Factors. 2015;33(1):8-13. doi:10.3109/08977194.2014.953630

12. Li R, Wu J, Lin Z, et al. Single injection of a novel nerve growth factor coacervate improves structural and functional regeneration after sciatic nerve injury in adult rats. Exp Neurol. 2017;288:1-10. doi:10.1016/j.expneurol.2016.10.015

13. Shakhbazau A, Kawasoe J, Hoyng SA, et al. Early regenerative effects of NGF-transduced Schwann cells in peripheral nerve repair. Mol Cell Neurosci. 2012;50(1):103-112. doi:10.1016/j.mcn.2012.04.004

14. Liu NK, Wang XF, Lu QB, Xu XM. Altered microRNA expression following traumatic spinal cord injury. Exp Neurol. 2009;219 (2):424-429. doi:10.1016/j.expneurol.2009.06.015

15. Eacker SM, Dawson TM, Dawson VL. Understanding microRNAs in neurodegeneration. Nat Rev Neurosci. 2009;10(12):837-841. doi: $10.1038 / \mathrm{nrn} 2726$

16. Zhou N, Hao S, Huang Z, et al. MiR-7 inhibited peripheral nerve injury repair by affecting neural stem cells migration and proliferation through cdc42. Mol Pain. 2018;14:1744806918766793. doi:10.1177/1744806918766793

17. Thornton JE, Gregory RI. How does Lin28 let-7 control development and disease? Trends Cell Biol. 2012;22(9):474-482. doi:10.1016/j. tcb.2012.06.001

18. Kucherenko MM, Barth J, Fiala A, Shcherbata HR. Steroid-induced microRNA let-7 acts as a spatio-temporal code for neuronal cell fate in the developing Drosophila brain. EMBO J. 2012;31 (24):4511-4523. doi:10.1038/emboj.2012.298

19. Lehmann SM, Kruger C, Park B, et al. An unconventional role for miRNA: let-7 activates Toll-like receptor 7 and causes neurodegeneration. Nat Neurosci. 2012;15(6):827-835. doi:10.1038/ nn. 3113

20. Zou Y, Chiu H, Zinovyeva A, Ambros V, Chuang CF, Chang C. Developmental decline in neuronal regeneration by the progressive change of two intrinsic timers. Science. 2013;340(6130):372-376. doi:10.1126/science. 1231321

21. Tanaka R, Fujita M, Tsuruta R, et al. Urinary trypsin inhibitor suppresses excessive generation of superoxide anion radical, systemic inflammation, oxidative stress, and endothelial injury in endotoxemic rats. Inflamm Res. 2010;59(8):597-606. doi:10.1007/s00011-010-0166-8

22. Wu Q, Liu LT, Wang XY, et al. Lycium barbarum polysaccharides attenuate kidney injury in septic rats by regulating Keap1-Nrf2/ARE pathway. Life Sci. 2020;242:117240. doi:10.1016/j.lfs.2019.117240 
23. Shu Y, Yang Y, Qiu W, et al. Neuroprotection by ulinastatin in experimental autoimmune encephalomyelitis. Neurochem Res. 2011;36(11):1969-1977. doi:10.1007/s11064-011-0520-4

24. Xie X, Li T, Yuan H. Protective effects of Ulinastatin on oxidative stress and inflammation of rat-derived cardiomyocytes H9c2. Am $J$ Transl Res. 2019;11(11):7094-7103.

25. Lin Y-F, Xie Z, Zhou J, Chen -H-H, Shao -W-W, Lin H-D. Effect of exogenous spastin combined with polyethylene glycol on sciatic nerve injury. Neural Regen Res. 2019;14(7):1271-1279. doi:10.4103/16735374.251336

26. Li S, Wang X, Gu Y, et al. Let-7 microRNAs regenerate peripheral nerve regeneration by targeting nerve growth factor. Mol Ther. 2015;23(3):423-433. doi:10.1038/mt.2014.220

27. Taylor CA, Braza D, Rice JB, Dillingham T. The incidence of peripheral nerve injury in extremity trauma. Am J Phys Med Rehabil. 2008;87(5):381-385. doi:10.1097/PHM.0b013e31815e6370

28. Shaw PX, Sang A, Wang Y, et al. Topical administration of a Rock/ Net inhibitor promotes retinal ganglion cell survival and axon regeneration after optic nerve injury. Exp Eye Res. 2017;158:33-42. doi:10.1016/j.exer.2016.07.006

29. Tamaddonfard E, Farshid AA, Samadi F, Eghdami K. Effect of vitamin B12 on functional recovery and histopathologic changes of tibial nerve-crushed rats. Drug Res. 2014;64(9):470-475. doi:10.1055/s-0033-1363219

30. Daeschler SC, Harhaus L, Bergmeister KD, et al. Clinically available low intensity ultrasound devices do not promote axonal regeneration after peripheral nerve surgery-A preclinical investigation of an FDA-approved device. Front Neurol. 2018;9:1057. doi:10.3389/ fneur.2018.01057

31. Atal SS, Atal S. Ulinastatin - a newer potential therapeutic option for multiple organ dysfunction syndrome. J Basic Clin Physiol Pharmacol. 2016;27(2):91-99. doi:10.1515/jbcpp-2015-0003

32. Chen P, Piao X, Bonaldo P. Role of macrophages in Wallerian degeneration and axonal regeneration after peripheral nerve injury. Acta Neuropathol. 2015;130(5):605-618. doi:10.1007/s00401-0151482-4

33. Flamm AG, Żerko S, Zawadzka-Kazimierczuk A, Koźmiński W, Konrat R, Coudevylle N. 1H, 15N, 13C resonance assignment of human GAP-43. Biomol NMR Assign. 2016;10(1):171-174. doi:10.1007/s12104-015-9660-9

34. Hung -C-C, Lin C-H, Chang H, et al. Astrocytic GAP43 induced by the TLR4/NF- $\kappa$ B/STAT3 axis attenuates astrogliosis-mediated microglial activation and neurotoxicity. J Neurosci. 2016;36(6):2027-2043. doi:10.1523/JNEUROSCI.3457-15.2016

35. Gentil BJ, Tibshirani M, Durham HD. Neurofilament dynamics and involvement in neurological disorders. Cell Tissue Res. 2015;360 (3):609-620. doi:10.1007/s00441-014-2082-7

36. Pronker MF, Lemstra S, Snijder J, et al. Structural basis of myelin-associated glycoprotein adhesion and signalling. Nat Commun. 2016;7(1):13584. doi:10.1038/ncomms13584

37. Tae HJ, Rahman MM, Park BY. Temporal and spatial expression analysis of peripheral myelin protein 22 (Pmp22) in developing Xenopus. Gene Expr Patterns. 2015;17(1):26-30. doi:10.1016/j. gep.2015.01.001

Drug Design, Development and Therapy

\section{Publish your work in this journal}

Drug Design, Development and Therapy is an international, peerreviewed open-access journal that spans the spectrum of drug design and development through to clinical applications. Clinical outcomes, patient safety, and programs for the development and effective, safe, and sustained use of medicines are a feature of the journal, which has also
38. Zhao HT, Damle S, Ikeda-Lee K, et al. PMP22 antisense oligonucleotides reverse Charcot-Marie-Tooth disease type 1A features in rodent models. J Clin Invest. 2018;128(1):359-368. doi:10.1172/JCI96499

39. Qiu J, Yang X, Wang L, et al. Isoquercitrin promotes peripheral nerve regeneration through inhibiting oxidative stress following sciatic crush injury in mice. Ann Transl Med. 2019;7(22):680. doi:10. 21037/atm.2019.11.18

40. Fineberg SK, Kosik KS, Davidson BL. MicroRNAs potentiate neural development. Neuron. 2009;64(3):303-309. doi:10.1016/j.neuron. 2009.10.020

41. Yoon AR, Gao R, Kaul Z, et al. MicroRNA-296 is enriched in cancer cells and downregulates p21WAF1 mRNA expression via interaction with its 3' untranslated region. Nucleic Acids Res. 2011;39 (18):8078-8091. doi:10.1093/nar/gkr492

42. Liu X, Cui X, Guan G, Dong Y, Zhang Z. microRNA-192-5p is involved in nerve repair in rats with peripheral nerve injury by regulating XIAP. Cell Cycle. 2020;19(3):326-338. doi:10.1080/ 15384101.2019.1710916

43. Gokbuget D, Pereira JA, Bachofner S, et al. The Lin28/let-7 axis is critical for myelination in the peripheral nervous system. Nat Commun. 2015;6(1):8584. doi:10.1038/ncomms9584

44. Shin YK, Jang SY, Yun SH, et al. Cooperative interaction of hepatocyte growth factor and neuregulin regulates Schwann cell migration and proliferation through Grb2-associated binder-2 in peripheral nerve repair. Glia. 2017;65(11):1794-1808. doi:10.1002/glia.23195

45. Lindborg JA, Mack M, Zigmond RE. Neutrophils are critical for myelin removal in a peripheral nerve injury model of Wallerian degeneration. J Neurosci. 2017;37(43):10258-10277. doi:10.1523/ JNEUROSCI.2085-17.2017

46. Shi G, Shi J, Liu K, et al. Increased miR-195 aggravates neuropathic pain by inhibiting autophagy following peripheral nerve injury. Glia. 2013;61(4):504-512. doi:10.1002/glia.22451

47. Komirishetty P, Areti A, Yerra VG, et al. PARP inhibition attenuates neuroinflammation and oxidative stress in chronic constriction injury induced peripheral neuropathy. Life Sci. 2016;150:50-60. doi:10.1016/j.lfs.2016.02.085

48. Lanza C, Raimondo S, Vergani L, et al. Expression of antioxidant molecules after peripheral nerve injury and regeneration. $J$ Neurosci Res. 2012;90(4):842-848. doi:10.1002/jnr.22778

49. Wang H, Ding XG, Li SW, et al. Role of oxidative stress in surgical cavernous nerve injury in a rat model. J Neurosci Res. 2015;93 (6):922-929. doi:10.1002/jnr.23545

50. Qian Y, Han Q, Zhao X, et al. 3D melatonin nerve scaffold reduces oxidative stress and inflammation and increases autophagy in peripheral nerve regeneration. $J$ Pineal Res. 2018;65(4):e12516. doi:10.1111/jpi.12516

51. Renno WM, Benov L, Khan KM. Possible role of antioxidative capacity of (-)-epigallocatechin-3-gallate treatment in morphological and neurobehavioral recovery after sciatic nerve crush injury. J Neurosurg Spine. 2017;27(5):593-613. doi:10.3171/2016.10.SPINE16218 been accepted for indexing on PubMed Central. The manuscript management system is completely online and includes a very quick and fair peer-review system, which is all easy to use. Visit http://www. dovepress.com/testimonials.php to read real quotes from published authors. 